Keywords: PTSD; Psychiatric emergencies;

Screening.

\title{
Screening for post-traumatic stress disorder (PTSD) in a psychiatric emergency setting
}

\section{Marie-Laure Ivanov ${ }^{\star}$ \\ Orest Weber* \\ Mehdi Gholam-Rezaee ${ }^{\star \star}$ \\ Gerrit Weber* \\ Daphné Reeves* \\ Rafik Benharrats* \\ Bertrand Yersin ${ }^{\star * *}$ \\ Friedrich Stiefel ${ }^{\star}$}

* Psychiatric liaison service, University Hospital Center and University of Lausanne, Lausanne

** Center of epidemiological psychiatry and psychopathology (CEPP), University Hospital Center and University of Lausanne, Prilly-Lausanne

*** Service of Emergency Medicine, University Hospital Center and University of Lausanne, Lausanne

SWITZERLAND

\begin{abstract}
Background and Objectives: (i) to assess the prevalence of PTSD in a psychiatric emergency setting by means of a diagnostic instrument and to compare it with PTSD-prevalence of a clinically evaluated, historical sample; and (ii) to assess psychiatric residents' perception of the systematic use of this diagnostic instrument.

Methods: A consecutive sample of patients $(\mathrm{N}=403)$ evaluated for a psychiatric emergency was assessed with the module J (PTSD) of the MINI, the historical sample ( $\mathrm{N}=350)$, assessed by chart review, consisted of consecutive patients of the same setting evaluated one year prior to the study period. Residents' perceptions were assessed by means of a focus group.

Results: While in only $0.57 \%$ of the historical sample $(\mathrm{N}=350)$ a diagnosis of PTSD was recorded, $20.3 \%(\mathrm{~N}=64)$ of the patients assessed with the diagnostic instrument $(\mathrm{N}=316)$ qualified for a diagnosis of PTSD. Higher prevalence rates were observed in refugees and those without legal residency status $(50 \%)$; patients from countries with a recent history of war $(47.1 \%)$; those with four $(44.4 \%)$ or three psychiatric co-morbidities $(35.3 \%)$; migrants $(29.8 \%)$ and patients without professional income (25\%). Residents felt that the systematic use of the tool was not adequate in the psychiatric emergency setting for various reasons (e.g.: not suitable for a first or single consultation, negative impact on the clinical evaluation).

Conclusions: The study confirms that PTSD is underdiagnosed in the psychiatric emergency setting. To improve the situation, targeted screening or educational and institutional strategies are needed.
\end{abstract}

Received: 1 April 2011

Revised: 20 January 2012

Accepted: 10 February 2012 


\section{Introduction}

Post-traumatic stress disorder (PTSD) is defined by the ICD-10 as an anxiety disorder in response to an extremely traumatic event $^{1}$. PTSD, associated in up to $80 \%$ of cases with psychiatric co-morbidities, such as mood disturbances or substance abuse ${ }^{2,3}$, is divided in the DSM-IVR-TR ${ }^{4}$ into an acute (symptoms for less than 3 months), chronic (symptoms for more than 3 months) and delayed type (symptoms emerge at least 6 months after exposition to the traumatic event). Lifetime prevalence of PTSD depends on populations studied and diagnostic criteria used $^{5}$. Screening for PTSD by means of diagnostic tools can be considered as meaningful in specific settings and patient populations, such as substance abusers ${ }^{6}$; patients with certain types of cancer ${ }^{7}$ or victims of various types of violence or accidents ${ }^{8-10}$, who are at risk of developping PTSD. Studies addressing PTSD specifically in the emergency setting are rare; we have found only two indicating a $13 \%$ prevalence of PTSD in Serbia after the war ${ }^{11}$ and a $14 \%$ prevalence among Albanians in Kosovo ${ }^{12}$. The first study assessed patients $(\mathrm{N}=562)$ presenting in general hospital emergency departments three years after the war in Serbia by means of a diagnostic tool and found overall prevalence of PTSD of $13 \%$, underlining a relatively high prevalence of PTSD in this patient population. The second study was also conducted in the emergency department of a general hospital in Pristina (Kosovo) and found a $14 \%$ prevalence of PTSD. In the first study, refugee status and social isolation, and in the second study the number of traumatic events, older age, being a woman and having less education were associated with PTSD.

Given these epidemiological data and the clinical impression that PTSD is underdiag- nosed in the psychiatric emergency setting, systematic evaluation of this population with a diagnostic tool could be an adequate strategy to improve detection rates.

The objectives of this study were (i) to identify the prevalence of PTSD in a psychiatric emergency setting by means of the systematic use of a diagnostic instrument, (ii) to compare this prevalence to the prevalence of an historical sample which was clinically evaluated and not assessed with a diagnostic tool, and (iii) to assess how the use of a diagnostic instrument for PTSD is perceived by clinicians working in this setting.

The study protocol was approved by the local ethics committee, who considered the use of a diagnostic instrument for PTSD as good medical practice and therefore decided that patients were to be informed orally about the study, but not asked to provide an informed consent.

\section{Methods}

Setting and residents: The study took place in the psychiatric liaison service, which is consulted by the patient or to which patients are referred by physicians or somatic colleagues of the hospital (see also patient population). The psychiatric liaison service, which encompasses the psychiatric emergency unit, is part of the general hospital and has an intensive collaboration with the general emergency service, since somaticians request consultations for patients with psychiatric co-morbidities and the psychiatric emergency unit transfers patients for somatic evaluations. A total of 16 psychiatric residents participated in the study. About one third $(\mathrm{N}=5)$ were trained in Switzerland, eight were trained in European 
countries and three in other countries (all of them speaking French adequately), with a majority $(\mathrm{N}=10)$ being native French-speaking. Professional experience varied between 6 months and 5 years, with a majority of residents $(\mathrm{N}=10)$ having been trained less than a year in psychiatry.

Training of residents: training how to use the diagnostic instrument was provided by the first author and consisted of a reminder of the diagnostic criteria of PTSD and a presentation of the diagnostic instrument. Training lasted one hour and was conducted in three different groups.

Assessment instrument: different diagnostic instruments for PTSD exist ${ }^{13}$; the decision to use the PTSD module of the Mini Mental Neuropsychologic Interview (MINI 5.0.0) was based on the following rationale: it was developed for clinical and research purposes; it is not time-consuming; it takes into account both DSM and ICD criteria and it has been translated into French ${ }^{14}$. The ICD10 French version of the PTSD module of the MINI used in this study (see Table $1^{15}$ ), is almost identical with the DSM IV PTSD module of the MINI ${ }^{14}$, but compared to DSM IV, PTSD can occur in ICD-10 after an unlimited time span and includes all types of PTSD. In addition, ICD-10 is the official medical coding system in Switzerland. The questionnaire consists of 3 screening questions, followed -if positively answered- by two diagnostic questions (see Table 1). The instrument was administered at the end of the consultation or during the consultation, if PTSD was an option to be explored by the resident. A diagnosis of PTSD is recorded if the question 4 or if at least 2 out of the 5 items of question 5 are answered positively. While this instrument has not been validated

Table 1

French version (2000) of the module PTSD of the MINI with 3 screening questions, followed - if positively answered - by 6 diagnostic questions. A diagnosis of PTSD is recorded if question 4 or if at least 2 out of the 5 items of question 5 are answered positively

1) Have you ever experienced, witnessed or faced an extremely traumatic event, during which people have died or you and / or other persons been in danger of being killed, were severely injured or were harmed with regard to their physical integrity? Examples of traumatic events: severe accident, physical assault, rape, terrorism, hostage-taking, kidnapping, fire, discovering a dead body, sudden death of a significant other, war, natural disasters...

2) Do you often think of this event in a distressing way, do you dream about it or do you frequently have the impression of re-experiencing it?

3) Since the occurrence of this event, have you had a tendency to avoid everything that could remind you of the event?

4) Do you have trouble recalling exactly what happened?

5) Since this event, have you noticed that you have changed, and do you currently:
a. have trouble falling asleep or wake up often?
b. seem especially irritable or have outbursts of anger?
c. have trouble concentrating?
d. feel nervous or constantly on your guard?
e. become easily startled? 
in its French version, it has been utilized in research ${ }^{15}$; the French version of the PTSD module of the MINI therefore seemed to be a good choice, given the fact that validation in another language is less important for a diagnostic instrument than for a psychometric instrument.

Patient populations: between December $1^{\text {st }} 2009$ and February $4^{\text {th }} 2010$, all consecutive patients, aged between 18 and 65 years, who consulted the psychiatric emergency unit of the Psychiatric Liaison Service or who were evaluated by a psychiatrist in the Service of Emergency Medicine of the University Hospital Lausanne (Switzerland) were included in the study. Given the fact that a growing number of migrants or patients unable to understand French consult the psychiatric and somatic emergency services, a translation by a hospital staff member or a specialised translation service is organized. Sex and age, date of inclusion in the study, place of birth, citizen status (Swiss citizen, residence permit, refugee or "illegal" immigrants) and profession (or source of financial support if without profession) were recorded.

Identification of recorded diagnosis of PTSD in the historical sample was based on a chart review by the first author of 350 consecutive patients consulting from December 2008 to the end of January 2009 in the same settings; these patients were not assessed with a diagnostic tool; diagnosis of PTSD was based on a clinical evaluation by the residents. The review of medical charts was effectuated by accessing the database of the psychiatric emergency unit, which contains administrative data, such as day of consultation, and psychiatric diagnoses recorded after the first consultation. This work was undertaken by the first author in the context of her doctoral dissertation in medicine. Given the fact that only sociodemographics and psychiatric diagnoses were identified, bias seemed not to be a problem.
Assessment of clinicians' perception of the use of a diagnostic tool: assessment of clinicians was based on a focus group. Focus groups are collective interviews based on interactive exchange of information by participants in order to analyze in depth their representations and experiences; focus groups are often used in medicine to investigate specific topics qualitatively ${ }^{16-18}$. The focus group of this study was conducted by a staff member of the psychiatric liaison service $(\mathrm{OW})$, who was not otherwise implicated in the study and who has extensive experience with qualitative research. After a brief introduction addressing the objectives and methods of the focus group, the psychiatric residents were invited to express their experiences with the use of the diagnostic instrument; two topics emerged and were further discussed in depth: (i) the clinical usefulness of the instrument and (ii) the impact of the instrument on the clinician-patient relationship.

Transcribed discussions were analysed independently by three of the investigators (DR, MLI, FS) who identified and labelled significant comments that described the informants' experiences (meaning units). After a mutual presentation and agreement on the meaning units, the first author under the supervision of the leader of the focus group classified the material and organised it into clusters of non-overlapping subthemes, which were validated by the investigators who initially identified and labelled significant comments. Finally, a scientific description was written which captured the essence of the participants' experiences.

Statistical analyses: the statistical analysis was made by using the SPSS 18 software. To test the association between two categorical variables, Pearson's chi-square test, which tests the null hypothesis assuming independence between categories of two 
variables, was used. In other words: for each test, the independence between two categorical variables was assessed. The restriction in this case is that the asymptotic chi-square law used in inference is not valid if at least $20 \%$ of the cells have an expected frequency smaller than five. In such cases, the Fisher exact test was used (instead of the Pearson's chi-square test) which is a non-parametric test and provides the exact probability of observing the observed cross-table under the hypothesis of independence among all possible tables with the same marginal frequencies.

\section{Results}

\section{Assessment of the prevalence of PTSD by means of a diagnostic tool}

Of the 403 consecutive patients, 87 (21.6\%) were excluded for the following reasons: the resident forgot to fill in the questionnaire $(\mathrm{N}$ $=46$ ), a verbal evaluation of the patient was not possible $(\mathrm{N}=22)$, patients refused to answer the questions $(\mathrm{N}=13)$ or data of the questionnaire was incomplete $(\mathrm{N}=6)$. Among the included patients, $58 \%(\mathrm{~N}=183)$ were women and $42 \%(\mathrm{~N}=133)$ were men, their ages ranged from 18 to 57 years. Born in 53 different countries, more than half of them ( $\mathrm{N}=169)$ were born in Switzerland, about one quarter $(\mathrm{N}=72)$ were born in a European country without a history of a recent war, in countries outside Europe (57) and a small minority in European countries with a recent war $(\mathrm{N}=17)$. More than half of them $(58.2 \%, \mathrm{~N}=184)$ had no professional activity. Almost two thirds $(64.9 \%, \mathrm{~N}=203)$ were Swiss citizens or in a stable living situation (long-term residency status); had an immigrant visa dependent on work $(26.8 \%$, $\mathrm{N}=84$ ), had refugee status or were "illegal" immigrants $(8.3 \%, \mathrm{~N}=26)$ (3 patients with missing data). Among the included patients $(\mathrm{N}=316), 64(20.3 \%)$ qualified for a diagnosis of PTSD.

With regard to the specific questions of the MINI (see Table 2), 138 patients (43.7\%) indicated having been exposed to an extremely traumatic event (question 1); 91 patients $(28.8 \%)$ reported remembering this event often in a distressing way, dreaming of it or having the impression of reexperiencing it frequently (question 2); 68 patients $(21.5 \%)$ confirmed a tendency to avoid anything which could remind them of the event (question 3). Almost all $(\mathrm{N}=64)$ of those who responded positively to these first three questions $(\mathrm{N}=68)$ qualified for a diagnosis of PTSD (by providing a positive answer to question 4 or to two items of question 5).

Of patients qualifying for PTSD according to the questionnaire, the diagnosis was documented in only one third $(\mathrm{N}=24)$ of their medical charts. In other words: for the majority of the cases, residents did diagnose but not document the disorder.

PTSD was always associated with at least one psychiatric co-morbidity. However, no specific ICD-10 diagnostic category was significantly more frequently associated with PTSD than another. The most frequent clinically identified psychiatric disorders of patients with PTSD were personality disorders of the emotionally labile type, depressive episodes and intoxications with psychotropics or analgesics.

The number of psychiatric co-morbidities correlated positively with a diagnosis of PTSD: $14.6 \%$ of patients with one psychiatric co-morbidity qualified for PTSD, as did $22 \%$ of those with two co-morbidities, $35.3 \%$ of those with three co-morbidities, and $44,4 \%$ of those with four co-morbidities. 
Table 2

Differences of overall-prevalence of PTSD and of answers to specific questions of the MINI are observed in sub-groups of the sample $(\mathrm{N}=316)$

\begin{tabular}{lll}
\hline Overall-Prevalence & $20.3 \%$ & $(\mathrm{~N}=64)$ \\
- Were exposed to a traumatic event (Q1) & $43.7 \%$ & $(\mathrm{~N}=138)$ \\
- Remember, dream of, reexperience (Q2) & $28.8 \%$ & $(\mathrm{~N}=91)$ \\
- Avoid (Q3) & $21.5 \%$ & $(\mathrm{~N}=68)$ \\
- Qualify for PTSD & $20.3 \%$ & $(\mathrm{~N}=64)$ \\
\hline Prevalence & & \\
- In men & $20.3 \%$ & $(\mathrm{~N}=27 / 133)$ \\
- In women & $20.2 \%$ & $(\mathrm{~N}=37 / 183)$ \\
- Born in Switzerland & $15.4 \%$ & $(\mathrm{~N}=26 / 169)$ \\
- Born in countries with a recent war & $47.1 \%$ & $(\mathrm{~N}=8 / 17) *$ \\
- Born in other countries & $29.8 \%$ & $(\mathrm{~N}=17 / 57) *$ \\
- Swiss nationality and residence permit & $17.8 \%$ & $(\mathrm{~N}=51 / 287)$ \\
- Refugee status or "illegal" migrants & $50.0 \%$ & $(\mathrm{~N}=13 / 26)^{* *}$ \\
- Currently employed & $13.6 \%$ & $(\mathrm{~N}=18 / 132) * * *$ \\
- Disabled, being on welfare or without financial support & $25.0 \%$ & $(\mathrm{~N}=46 / 184)$ \\
\hline * $<$ *0.05 & & $* * * \mathrm{P}=0.013$ \\
\hline
\end{tabular}

P-values correspond to Pearson's chi-square tests of association or Fisher exact tests if necessary.

No significant correlations between PTSD and age or sex were observed; 37 of the 183 women $(20.2 \%)$ and 27 of the 133 men $(20.3 \%)$ were diagnosed with PTSD. Prevalence of PTSD among patients born in Switzerland was $15.4 \%(\mathrm{~N}=26 / 169)$; patients born in other countries $(\mathrm{N}=17 / 57$, $29.8 \%$ ) and in countries with a recent history of war $(\mathrm{N}=8 / 17,47.1 \%)$ showed higher $(\mathrm{p}=$ 0.005) prevalence of PTSD (see Table 2).

Comparison of patients with Swiss nationality and those with a residence permit (stable situation) $(\mathrm{N}=51 / 287,17.8 \%)$ versus patients with refugee status or "illegal" immigrants $(\mathrm{N}=13 / 26,50 \%)$ revealed that prevalence of PTSD was significantly higher $(p<0.001)$ in the second group (see Table 2).
Patients without a professional income (disability, being on social welfare or without financial resources) also had significantly higher prevalence rates of PTSD $(\mathrm{N}=$ $46 / 184,25.0 \%)$ than those with a job $(\mathrm{N}=$ 18/132, 13.6\%); $(\mathrm{p}=0.013)$ (see Table 2).

\section{Comparison with the prevalence of PTSD in the historial sample}

Identification of a recorded diagnosis of PTSD on the charts of the historical sample $(\mathrm{N}=350)$ revealed only 2 cases of PTSD $(0.57 \%)$. Given the small numbers of PTSD cases, the sample was extended to a one year period, confirming again a prevalence of recorded diagnoses of PTSD below $1 \%$ (21/2983 patients, $0.75 \%)$. 


\section{Residents' perception of the diagnostic tool}

The focus group was conducted with 8 psychiatric residents who participated in the study, most of whom (5/8) were at the beginning of their training in psychiatry. The focus group lasted about one hour until the general impression emerged that "all of what had to be said has been said".

The following categories and specific topics were indentified: (i) use of the questionnaire, (ii) influence of the questionnaire on the psychiatric interview and (iii) impact on the therapeutic process. This last category was subdivided into two specific topics (a) impact on the clinical evaluation and (b) on the therapeutic relationship.

With regard to the first category "use of the instrument", most of the participants indicated that after a while the questionnaire had become "comfortable" and "easy" to use $(6 / 8) ; 6 / 8$ participants reported that after the study period, they still integrate the questions of the instrument in many of their interviews, but mainly (5/8) during followup and not first interviews.

With regard to the second category "the influence of the questionnaire on the psychiatric interview", all participants considered that the questionnaire should not be used systematically in the psychiatric emergency setting for the following reasons: the aim of some consultations is sometimes specific (e.g. request for a sick-leave); the time of the intervention is short; most patients are only seen once; some patients are unable to answer and questioning the presence of PTSD may be very time-consuming.

With regard to the first topic of the third category "the impact of the questionnaire on the clinical evaluation", all clinicians perceived the "mandatory" use of the question- naire as a constraint and inappropriate, contaminating the evaluation ("as if we were only focusing on PTSD"); however, some (3/8) also felt that the questionnaire helped ensure that questions about traumatic events were not avoided. Half of them (4/8) indicated that they integrated the questionnaire somehow into their clinical interview and did not use it just as a "question and answer" tool.

With regard to the second topic of the third category "the impact of the questionnaire on the therapeutic relationship", participants disagreed: $2 / 8$ indicated a negative impact, while $4 / 8$ felt that any question allowing a better understanding of the patient, especially in the emergency setting, may foster the therapeutic relationship. The issue of if and how the information obtained is transmitted, for example to the treating physician of the patient, was also considered as a problem by some of the participants (3/8). Finally, participants emphasized that the questionnaire revealed very complex problems, difficult to handle for both the patient and the clinician, or, as a participant stated: "And what do we do with this information in an initial psychiatric consultation, which often has no follow-up...?".

\section{Discussion}

Systematic use of a diagnostic instrument identified 64 out of 316 psychiatric emergency patients $(20.3 \%)$ with a diagnosis of PTSD and confirmed that this disorder is underdiagnosed in this setting. With regard to the specific questions of the instrument, one observes that with the first three screening questions the overwhelming majority of patients with PTSD were detected. The fact that patients answered by different percentages to questions 1 and 2 and 3 (see Table 1) 
illustrate that symptom expression of PTSD may vary, calling for a careful evaluation of all symptoms with diagnostic relevance. However, despite a positive result with the questionnaire, diagnosis of PTSD was recorded on the medical chart in only $1 / 3$ of the cases, which may also explain the low prevalence (less than $1 \%$ ) found in the chart review of the historical sample.

No single psychiatric diagnosis was significantly associated with PTSD; since no diagnostic screening for psychiatric diagnoses was effectuated, this result may not be reliable: substance abuse, for example, is strongly associated with PTSD, but was not observed more frequently than other diagnoses. However, the number of psychiatric co-morbidities increased the probability of being diagnosed with PTSD, a fact which had been observed already for patients presenting in emergency departments suffering from PTSD ${ }^{11}$. As there is doubt among clinicians that systematic screening for PTSD is appropriate in the psychiatric emergency setting, an alternative could be to target screening on patients with multiple psychiatric co-morbidities, and on those groups showing higher prevalence of PTSD, such as migrants from countries with a recent war, patients without legal residency status, the disabled and those without income. In addition to coming from regions which were exposed to war, less education, social isolation and refugee status have been associated with PTSD in patients presenting an emergency department ${ }^{11,12}$.

Indeed, psychiatric residents said that they got accustomed to using a diagnostic tool systematically and then integrated these questions into the patient interview after the end of the study. However, they also emphasized that screening was inappropriate in a first interview without follow-up. They also considered that using the tool had some- times had a negative impact on the interview and the therapeutic relationship. This raises the question of whether screening for PTSD (and other psychiatric diagnoses) is feasible in the psychiatric emergency setting or if other strategies, such as targeted screening of patients at risk (e.g. patients with a refugee status or those being on social welfare or with multiple psychiatric co-morbidities) or repeated teaching about PTSD may be a more efficient strategy to improve the situation. The fact that residents had to use the diagnostic instrument for the study period can be considered as training, since many went on using these questions after termination of the study. This illustrates that transfer of knowledge may be relatively easy to achieve. Despite the fact that patients with PTSD often find it difficult to access care and frequently consult emergency settings offering no follow-up after the consultation $^{11,12}$, up to now, no study has evaluated the effectiveness of different approaches to improve detection rates. It is not possible to know if the patient population of this study is similar to that presenting in psychiatric emergency settings of other European countries; the fact that the prevalence of migrants, for example, can differ and that the background and "local culture" of psychiatric residents differ, makes it difficult to generalize the results of this study.

This study also confirms that systematic screening for psychiatric disorders is difficult to implement ${ }^{19,20}$. This finding, together with the very low rates of diagnosed PTSD recorded on the medical chart also raises the question of what kind of institutional strategies have to be undertaken to improve the situation. While there are many studies evaluating specific diagnostic and therapeutic strategies for psychiatric disorders, less scientific effort is focused on "real world situations", indicating an important gap between 
what is known and could be done for psychiatric patients and what is finally done in daily clinical practice. In order to translate effectively the growing body of evidence into daily clinical practice, different educational strategies and systemic interventions on the setting will have to be conceptualized and evaluated ${ }^{21}$. However, the emergency setting is associated with an increased use by patients with PTSD and management strategies should therefore be developed ${ }^{22}$.

In conclusion, the first objectives of the study, to assess prevalence of PTSD identified by means of a sample reveals that PTSD is most probably underdiagnosed in daily clinical practice. The systematic use of a diagnostic instrument, however, is rather negatively perceived by the residents (second objective) indicating that probably only targeted screening of patients at risk or other strategies to improve detection of PTSD seems to be realistic options. While the results concerning the first objective might be generalizable to different services in different countries, the results concerning the implementation of a diagnostic tool in daily clinical practice will depend on local characteristics of the setting.

The study has several limitations. Among them, and most important, it remains unknown what really happened during the interviews: if and how PTSD was discussed with the patients, when identified, is more important than if PTSD was recorded on the medical chart. Nor did the study deal with the question of how patients perceived the diagnostic tool or if a targeted screening of patients at risk would have been approved by the residents. Finally, that fact that the French translation of the PTSD module of the MINI used in this study has not been validated also represents a limitation.

\section{References}

1. World Health Organisation. The ICD-10 Classification of Mental and Behavioural disorders: Clinical descriptions and diagnosis guidelines. Geneva: World Health Organisation; Chapter V (F 43.1); 1993.

2, Grinage BD. Diagnosis and Management of Posttraumatic Stress Disorder. Am Fam Physician 2003; 68: 2401-2408.

3. Kessler RC, Sonnega A, Bromet E, Hughes M, Nelson CB. Posttraumatic stress disorder in the National Comorbidity Survey. Arch Gen Psychatry 1995; 52: 1048-1060.

4. American Psychiatric Association. Diagnostic and Statistical Manual of Mental Disorders, forth edition, text revision, DSM-IVR-TR. Washington, DC: American Psychiatric Association; 2000. p. 467-468.

5. Kessler RC, Berlung P, Demler O, Jin R, Merikangas KR, Walters EE. Lifetime Prevalence and Age-of-Onset Distributions of DSM-IV Disorders in the National Comorbidity Survey Replication. Arch Gen Psychiatry 2005; 62: 593-602.

6. van Dam D, Ehring T, Vedel E, Emmelkamp PM. Validation of the Primary Care Posttraumatic Stress Disorder screening questionnaire (PC-PTSD) in civilian substance use disorder patients. J Subst Abuse Treat 2010; 39(2): 105-113.

7. Poluszny DM, Edwards RP, Dew MA, Baum A. Perceived threat and PTSD symptoms in women undergoing surgery for gynecologic cancer or benign conditions. Psycho-Oncology 2011; 20(7): 783-787.

8. Fraguas D, Terán S, Conejo-Galindo J, Medina O, Sainz Cortón E, Ferrando L, et al. Posttraumatic stress disorder in victims of the March 11 attacks in Madrid admitted to a hospital emergency room: 6-month follow-up. Eur Psychiatry 2006; 21(3): 143-151.

9. Breslau N. Epidemiologic studies of trauma, posttraumatic stress disorder and other psychatric disorders. Can J Psychiatry 2002; 47(10): 923-929.

10. Kessler RC, Üstün T. The WHO World Mental Health Surveys: Global perspectives on the epidemiology of mental disorders. New York: Cambridge University Press; 2008. p. $1-580$.

11. Nelson BD, Fernandez WG, Galea S, Sisco S, Dierberg K, Gorgieva GS, et al. War-related psychological sequelae among emergency department patients in the former Republic of Yugoslavia. BMC Med 2004; 2-22.

12. Fernandez WG, Galea S, Ahern J, Sisco S, Waldman RJ, Koci B, et al. Mental health status among ethnic Albanians seeking medical care in an emergency department two years after the war in Kosovo: A pilot project. Ann Emerg 2004; 43(2): 1-8. 
13. Brewin CR. Systematic review of screening instruments for adults at risk of PTSD. J Trauma Stress 2005; 18 : 53-62.

14. Sheehan DV, Lecrubier Y, Sheehan KH, Amorim P, Janavs J, Weiller E, et al. The Mini-International Neuropsychiatric Interview (M.I.N.I): The development and validation of a structured diagnostic psychiatric interview for DSM-IV and ICD 10. J Clin Psychiatry 1998; 59 (suppl 20): 22-33.

15. Lecrubier Y, Weiller E, Hergueta T, Amorim P, Bonora LI, Lépine JP. Mini International Neuropsychiatric Interview. 2000; Version ICD-10 INSERM-Paris, France, January 3 .

16. Moreau A, Dedianne MC, Le Goaziou MF, Labarère J, Terra JL. Recherche en médecine générale, s'approprier la méthode du focus groupe. La Revue du Praticien Médecine Générale 2004; 18(645): 382-384.

17. Morgan D. Focus groups as qualitative research. Thousand Oaks: Sage; 1997.

18. Puchta C, Potter J. Focus group practice. Thousand Oaks: Sage; 2004.

19. Michaud L, Büla C, Berney A, Camus V, Voellinger R, Stiefel F, et al. Delirium: Guidelines for general hospitals. Psychosom Res 2007; 62: 371-383.
20. Michaud L, Voellinger R, Burnand B, Stiefel F. Major depressive disorders in the general hospital: How to implement guidelines. Psychosom Res 2006; 60: 455-459.

21. Stiefel FC, Zdrojewski C, Bel Hadj F, Boffa D, Dorogi Y, So A, et al. Effects of a Multifaceted Psychiatric Intervention Targeted for the Complex Medically ill: a Randomized Controlled Trial. Psychother Psychosom 2008; 77: 247-256.

22. Fogarty CT, Sharma S, Chetty VK, Culpepper L. Mental Health Conditions are Associated With Increased Health Care Utilization Among Urban Family Medicine Patients. J Am Board Fam Med 2008; 21: 398-407.

Author for correspondence:

Prof. F. Stiefel

Psychiatric liaison service

University Hospital Center and University of Lausanne

Rue du Bugnon 44, 1011 Lausanne

Switzerland

Phone 0041-21-314 1090

Fax 0041-21-314 1086

E-mail: Frederic.Stiefel@chuv.ch 